\title{
Nicotine and Non-Nicotine Smoking Factors Differentially Modulate Craving, Withdrawal and Cerebral Blood Flow as Measured with Arterial Spin Labeling
}

\author{
Merideth A Addicott ${ }^{1,2}$, Brett Froeliger ${ }^{3,4}$, Rachel V Kozink', Dana M Van Wert', Eric C Westman ${ }^{5}$, \\ Jed E Rose' and Francis J McClernon*,I,2 \\ 'Department of Psychiatry and Behavioral Sciences, Duke University Medical Center, Durham, NC, USA; ${ }^{2}$ Duke-UNC Brain Imaging and Analysis \\ Center, Duke University Medical Center, Durham, NC, USA; 'Department of Neurosciences, Medical University of South Carolina, Charleston, SC, \\ USA; ${ }^{4}$ Hollings Cancer Center, Charleston, SC, USA; ${ }^{5}$ Department of Medicine, Duke University Medical Center, Durham, NC, USA
}

Smoking cessation results in withdrawal symptoms such as craving and negative mood that may contribute to lapse and relapse. Little is known regarding whether these symptoms are associated with the nicotine or non-nicotine components of cigarette smoke. Using arterial spin labeling, we measured resting-state cerebral blood flow (CBF) in 29 adult smokers across four conditions: ( 1 ) nicotine patch + denicotinized cigarette smoking, (2) nicotine patch + abstinence from smoking, (3) placebo patch + denicotinized cigarette smoking, and (4) placebo patch + abstinence from smoking. We found that changes in self-reported craving positively correlated with changes in CBF from the denicotinized cigarette smoking conditions to the abstinent conditions. These correlations were found in several regions throughout the brain. Self-reported craving also increased from the nicotine to the placebo conditions, but had a minimal relationship with changes in CBF. The results of this study suggest that the non-nicotine components of cigarette smoke significantly impact withdrawal symptoms and associated brain areas, independently of the effects of nicotine. As such, the effects of non-nicotine factors are important to consider in the design and development of smoking cessation interventions and tobacco regulation. Neuropsychopharmacology (2014) 39, 2750-2759; doi:I0.1038/npp.2014.108; published online I3 August 20I4

\section{INTRODUCTION}

Chronic tobacco smoking leads to changes in brain physiology, such as an upregulation of nicotinic acetylcholine receptors (nAChRs) (Watkins et al, 2000), and tobacco withdrawal is marked by symptoms, such as anxious and irritable mood, craving, and difficulty concentrating (APA, 2000; Hughes and Hatsukami, 1986). Among habitual smokers, these symptoms may begin within an hour after the last cigarette (Hendricks et al, 2006) and can last for weeks (Gilbert et al, 1998, 2002). The subjective craving phenomenon is not trivial-it predicts relapse following a quit attempt (Killen and Fortmann, 1997)_although little is known regarding the links between craving, withdrawal and brain physiology, or the differential influence of nicotine and non-nicotine factors on these phenomena.

*Correspondence: Dr FJ McClernon, Department of Psychiatry and Behavioral Sciences, Duke University, 2608 Erwin Rd, Box 3527, Lakeview Pavilion E, suite 300, Durham, NC 27705, USA, Tel: +919684 5237, Fax: +9196810016,

E-mail: francis.mcclernon@dm.duke.edu

Received 30 December 2013; revised 8 April 20।4; accepted 10 April 2014; accepted article preview online 13 May 2014

\section{Nicotine and non-nicotine factors in smoking}

Although nicotine is the most studied psychoactive constituent of tobacco smoke, non-nicotine factors are believed to contribute to smoking reward and reinforcement as well (Rose, 2006). More than 5000 components have been identified in tobacco smoke (Rodgman and Perfetti, 2013), and some of these components have psychoactive effects that may contribute to tobacco's addiction liability (Talhout et al, 2007). Moreover, the sensorimotor effects of smoking cigarettes are known to have a critical influence on the subjective experience of smoking, and these effects may be an essential element of cue-conditioning and the persistence of craving. Although many of the sensory effects of smoking (eg, taste, aroma, impact on throat) can be irritating at first, they may become reinforcing as they are repeatedly paired with nicotine (Bevins and Palmatier, 2004; Rose and Levin, 1991). Indeed, smokers are sensitive to changes or blockade of these sensations (McClernon et al, 2007; Perkins et al, 2001; Rose et al, 1990) and cite them as a motive for continued smoking (Piper et al, 2008). Smokers also report that they miss the act of smoking a cigarette following cessation (Rose et al, 1990). Consistent with the above, denicotinized cigarettes, in which the sensorimotor and non-nicotine psychoactive components of smoking are experienced in the absence of nicotine, have 
been shown to suppress withdrawal symptoms (eg, irritability and anxiety) (Gross et al, 1997; Pickworth et al, 1999) and craving (Buchhalter et al, 2005; Rose and Behm, 2004). For example, smokers reported a reduction in cravings after smoking denicotinized cigarettes and separately after an infusion of intravenous nicotine; however, denicotinized cigarettes produced a pleasurable subjective experience, whereas nicotine alone did not (Rose et al, 2000). Furthermore, smoking behavior among habitual smokers can be maintained by denicotinized cigarettes alone (Donny et al, 2007).

\section{Nicotine, Smoking, and Cerebral Blood Flow}

A number of previous studies have investigated the effects of nicotine, smoking and smoking withdrawal on regional cerebral blood flow (rCBF). Nicotine and tobacco smoke have both vasoconstrictive and vasodilatory effects on the cerebrovasculature (Iida et al, 1998). Long-term cigarette smoking has been shown to reduce rCBF (Rogers et al, 1985; Yamashita et al, 1988); however, overnight abstention from cigarettes among chronic smokers has been shown to have limited (Wang et al, 2007) or no effect on rCBF (Shinohara et al, 2006; Tanabe et al, 2008). Other studies have shown both increases and/or decreases in rCBF owing to nicotine administration or cigarette smoking (Mathew and Wilson, 1991; Domino et al, 2000, 2004; Rose et al, 2003); some of these differences may depend on whether smokers were tested in an abstinent or satiated state (Zubieta et al, 2005; Tanabe et al, 2008) or on the measures used to quantify $\mathrm{rCBF}$ (eg, magnetic resonance imaging $v s$ positron emission tomography).

Although the effects of nicotine and cigarette smoke on rCBF have been somewhat inconsistent, studies have more reliably found correlations between subjective reports of withdrawal symptoms (including craving) and changes in rCBF (Franklin et al, 2007; Rose et al, 2003; Tanabe et al, 2008; Wang et al, 2007). Resting-state rCBF may indirectly reflect neural activity or synaptic function related to tobacco addiction and craving. Studies measuring $\mathrm{rCBF}$ have shown positive correlations with craving in regions including the pons, midbrain, thalamus, and occipital cortex (Rose et al, 2003), as well as the posterior cingulate and dorsolateral prefrontal cortices (Franklin et al, 2007). Specifically, a positive correlation between $\mathrm{rCBF}$ in the anterior cingulate and hippocampus with changes in craving scores was reported before and after smoking the first cigarette of the day (Zubieta et al, 2005). Increased craving also predicted increased $\mathrm{rCBF}$ from smoking satiety to abstinent conditions in regions including the frontal and occipital lobes, striatum and thalamus (Wang et al, 2007), suggesting increased neural activity in these regions underlies abstinence-induced cravings. Nicotine, in the absence of smoking, has been shown to produce a negative correlation between a change in reported withdrawal symptoms and a change in thalamic cerebral blood flow (CBF) in overnight withdrawn smokers (Tanabe et al, 2008). Although these and other studies suggest a relationship between smoking, withdrawal symptoms, and CBF, it is still unclear whether this relationship is moderated by nicotine or by the non-nicotine components of cigarette smoke.

\section{The Present Study}

Over the last decade, there has been a growing appreciation of the importance of the sensorimotor and non-nicotine psychoactive components of cigarette smoking in smoking initiation, maintenance, and relapse. However, very little work has been conducted to specifically evaluate the neurobiological basis of differences in the effects of nicotine and non-nicotine factors. The current study was designed to investigate the main and interactive effects of nicotine and non-nicotine components of smoking. The study also addressed whether the change in nicotine levels or nonnicotine components of cigarette smoke affected the relationship between craving and CBF. Smokers were scanned on four separate occasions using a factorial design: following $24 \mathrm{~h}$ of wearing either a $21 \mathrm{mg} /$ day nicotine or placebo patch combined with $24 \mathrm{~h}$ of smoking denicotinized cigarettes or remaining smoking abstinent. Craving and other withdrawal symptoms, as well as CBF, were measured in each of the four conditions. A control group of nonsmokers were scanned once and provided a comparison group for validating our assessment of global $\mathrm{CBF}$.

\section{MATERIALS AND METHODS}

\section{Participants}

Thirty-five smokers, aged 18-55 years, were recruited from the community and completed all aspects of the study. Smokers were included in the study if they were generally healthy, right-handed, smoked $\geqslant 10$ cigarettes/day of a brand delivering $>0.5 \mathrm{mg}$ of nicotine according to the standard Federal Trade Commission method for at least 2 years, were not using any nicotine products other than cigarettes, and had no desire to quit smoking while participating in the study. Smokers were required to have an expired $\mathrm{CO}$ concentration of $\geqslant 10 \mathrm{ppm}$ or $100 \mathrm{ng} / \mathrm{ml}$ or greater of urinary cotinine (Nicalert) on the day of the screening visit. Exclusionary criteria included serious health problems (eg, hypertension), currently undergoing treatment for psychiatric illness, taking any medication that affects the central nervous system, any conditions that would make MRI unsafe, having a positive urine drug screen, and having a positive serum pregnancy test (for women).

An age-, race-, and sex-matched sample of nonsmokers was also recruited for comparison. The nonsmokers had the same inclusion/exclusion criteria, except that nonsmokers were required to have smoked $<50$ cigarettes in their lifetime, and have an expired CO concentration of $\leqslant 5 \mathrm{ppm}$ and less than $100 \mathrm{ng} / \mathrm{ml}$ of urinary cotinine (Nicalert) on the screening visit.

All participants read and signed a consent form prior to participation. All procedures were approved by the Duke University Medical Center Institutional Review Board. Participants completed a screening session where they provided breath and urine samples, underwent a medical evaluation, and completed questionnaires regarding smoking history and suitability for MRI research. Using a withinsubjects design, smokers underwent four imaging sessions at least 4 days apart. For $24 \mathrm{~h}$ prior to each session, smokers wore either a nicotine (21 mg Nicoderm CQ) or placebo patch, and either smoked denicotinized cigarettes (Quest 3, 
Vector Tobacco Co.) or abstained from smoking. Abstinence was verified by expired $\mathrm{CO}$ on the scan day. Patches were repackaged and were administered double-blind. Denicotinized cigarettes were mentholated or non-mentholated according to the participant's preference. During the denicotinized cigarette condition, participants were instructed to smoke as usual in the $24 \mathrm{~h}$ up to and immediately before the scan; participants were aware the cigarettes were denicotinized. There were four conditions in a $2(\mathrm{PATCH}) \times 2$ (SMOKE) design: (1) nicotine patch + denicotinized cigarette smoking (NIC/DENIC), (2) nicotine patch + abstinent from smoking (NIC/ABST), (3) placebo patch + denicotinized cigarette smoking (PLA/DENIC), and (4) placebo patch + abstinent from smoking (PLA/ABST). Nonsmokers were scanned on a single occasion.

\section{Questionnaires}

Smokers completed the Fagerstrom Test for Nicotine Dependence (FTND) (Heatherton et al, 1991) at screening and a modified version of the Shiffman-Jarvik Withdrawal Questionnaire (SJWQ; (Shiffman and Jarvik, 1976) to measure craving and withdrawal symptoms at the beginning of each scanning session. A 32-item measure, the SJWQ contains six subscales and asks the individual to evaluate on a scale of 1-7 the extent to which she/he agrees with each question regarding craving (eg, Are you thinking of cigarettes?), negative affect (eg, Do you feel irritable?), appetite (eg, Do you feel hungrier than usual?), arousal (eg, Are you able to concentrate?), somatosensory symptoms (eg, Do you have an upset stomach?), and habit withdrawal (eg, Do you miss having something to do with your hands?).

\section{Imaging}

Scans were conducted on a $3.0 \mathrm{~T}$ GE EXCITE HD scanner equipped with $40 \mathrm{mT} / \mathrm{m}$ gradients (Waukesha, WI). Pulsed arterial spin labeling (pASL) was used to measure CBF with a modified flow-sensitive alternating inversion recovery sequence (Kim, 1995) using QUIPSS II saturation pulses (Wong et al, 1998). This technique provides a quantitative measure of CBF by magnetically tagging arterial blood and measuring the flow of this blood into the imaging slice, whereas minimizing the problem of the variable transit delay between the arterial tag and the entry of the blood into the imaged slice (Wong et al, 1998). After localizer images were obtained, 53 resting label/control image pairs were obtained using the following parameters: $\mathrm{TR}=4000 \mathrm{msec}$, $\mathrm{TE}=3 \mathrm{msec}$, TI $1=600 \mathrm{msec}$, TI $2=1600$, field of view $=220$ $\times 220 \mathrm{~mm}$, acquisition matrix $=64 \times 64$, flip angle $=90$ degrees, 24 slices with $1-\mathrm{mm}$ slice gap acquired inferior to superior, original voxel size $=3 \times 3 \times 5 \mathrm{~mm}$.

During the scan, participants rested with eyes open. Alertness was monitored via a camera attached to the headcoil. Following the ASL sequence were blood oxygen level-dependent functional imaging runs, the data from which will be presented elsewhere. A high-resolution anatomical scan was acquired last using a 3D fast spoiled gradient echo (FSPGR) sequence with the following parameters: $\mathrm{TR}=7.292 \mathrm{msec}, \mathrm{TE}=2.952 \mathrm{msec}, \mathrm{TI}=450 \mathrm{msec}$, field of view $=256 \times 256 \mathrm{~mm}$, acquisition matrix $=256$ $\times 256$, flip angle $=25$ degrees.
CBF images were realigned and motion corrected using AFNI software (http://afni.nimh.nih.gov/), then converted into quantitative maps using a method described in (Wong et al, 1998) and with a script provided by the Cerebral Blood Flow Biomedical Informatics Research Network (https://cbfbirn.ucsd.edu/). Subsequently, images were coregistered and normalized to an MNI template then smoothed with an 8-mm Gaussian filter using SPM8 (http:// www.fil.ion.ucl.ac.uk/spm/software/spm8/).

To extract average gray matter CBF (gCBF), the MNI template was segmented using fslmaths (http://fsl.fmrib.ox.ac.uk/fsl/fslwiki/) to create a mask of voxels with a $51 \%$ probability of gray matter. This mask was applied to each individual's smoothed CBF image and the average voxel value in $\mathrm{ml}$ blood $/ 100 \mathrm{~g}$ tissue/min was quantified using fslstats (excluding voxels with zero values) (http://fsl.fmrib. ox.ac.uk/fsl/fslwiki/).

\section{Data Analysis}

Participant demographics were explored using $\chi^{2}$ and independent samples $t$-test. CO levels and self-reported withdrawal symptoms were investigated separately using 2 $(\mathrm{PATCH}) \times 2(\mathrm{SMOKE})$ analyses of variance (ANOVA). An exploratory analysis of sex differences in self-reported withdrawal symptoms revealed no significant effects; thus, sex was not included as a variable of interest in further analyses. Changes in whole-brain $\mathrm{gCBF}$ within smokers were analyzed using a $2(\mathrm{PATCH}) \times 2(\mathrm{SMOKE})$ ANOVA. Differences between smokers and nonsmokers were analyzed using a univariate ANOVA. Results were considered significant using a criterion alpha (two-tailed) $=0.05$.

Regional differences in $\mathrm{CBF}$ ( $\mathrm{rCBF}$ ) were investigated using a $2(\mathrm{PATCH}) \times 2(\mathrm{SMOKE})$ ANOVA and regression analyses. The change in $\mathrm{CO}$ levels between conditions was used as a covariate of no interest in the $\mathrm{rCBF}$ regression analyses in order to control for the vasodilatory effects of CO (Sinha et al, 1991). Lastly, we investigated the relationship between changes in withdrawal symptoms and changes in $\mathrm{rCBF}$. For both the self-report symptoms and the rCBF maps, change scores for the PATCH effect were calculated by taking the average of the two placebo conditions (PLA/ DENIC + PLA/ABST) and subtracting the average of the two nicotine conditions (NIC/DENIC + NIC/ABST). Similarly, change scores for the SMOKE effect were calculated by taking the average of the two ABST conditions (PLA/ ABST + NIC/ABST) and subtracting the average of the two DENIC smoking conditions (PLA/DENIC + NIC/DENIC). The change in the subscales craving, habit withdrawal, and negative affect were entered into multiple regression analyses with the change in $\mathrm{rCBF}$ and separately with the change in $\mathrm{gCBF}$, controlling for sex and changes in $\mathrm{CO}$ levels. Main effects and regression analyses for $\mathrm{rCBF}$ were considered significant if they passed a statistical threshold of $p<0.05$ cluster corrected. Cluster size for the comparisons was determined using AlphaSim and running Monte Carlo simulations (Ward, 2000) $(p<0.005$, uncorrected; $\geqslant 157$-voxel cluster of contiguous significant voxels).

Out of 35 smokers, 1 participant was removed for not following the request to abstain from smoking during the no smoking conditions (based on CO levels on the study day), 2 participants were removed for excessive motion on 
one or more study day ( $>6 \mathrm{~mm}$ ), and 4 participants were removed for hyperintensities or artifacts in their $\mathrm{CBF}$ images. Thus, 9 smokers (116 scans) were included in the data analysis. Out of 32 nonsmokers, 3 participants were removed for hyperintensities or artifacts on their CBF images. Twenty-nine nonsmokers were included in the data analysis.

\section{RESULTS}

\section{Participants}

The sample of smokers $(n=29,14$ women $)$ was (mean \pm SD) $38 \pm 10$ years of age and consisted of 14 Caucasians, 1 Asian, and 14 African-Americans. Smokers had an FTND score of $5.2 \pm 2.2$ and smoked $17 \pm 5$ cigarettes per day for the past $21 \pm 10$ years. The nonsmoker sample ( $n=29,11$ women) was $37 \pm 11$ years of age and consisted of 18 Caucasians, 1 Asian, and 10 African-Americans. There were no significant differences in age, sex, or racial distribution between the smokers and nonsmokers.

\section{CO Level}

As expected, smoking denicotinized cigarettes was associated with higher CO levels compared with abstinence (main effect of SMOKE: $15 \pm 1$ vs $4 \pm 2$ ppm, $\mathrm{F}(1,28)=43.1$, $p<0.001)$. There was no effect of PATCH on CO levels.

\section{Withdrawal Symptoms}

Smokers reported more withdrawal symptoms in the ABST condition than in the DENIC condition. These main effects of SMOKE were observed for self-reported craving $(\mathrm{F}(1,28)=$ $12.38, p=0.002)$, negative affect $(\mathrm{F}(1,28)=18.65, p<0.001)$, and habit withdrawal $(\mathrm{F}(1,28)=25.63, p<0.001)$; see Figure 1. Likewise, smokers reported more withdrawal symptoms in the PLA condition than in the NIC condition resulting in less robust but still significant main effects of PATCH for these same subscales: craving $(\mathrm{F}(1,28)=6.08$, $p=0.020)$, negative affect $(\mathrm{F}(1,28)=5.15, p=0.031)$, and habit withdrawal $(\mathrm{F}(1,28)=4.26, p=0.048)$. No interactions between PATCH and SMOKE were observed for any withdrawal symptom scales.

\section{gCBF}

Consistent with previous literature (Gur and Gur, 1990), female smokers had greater gCBF than male smokers $(42.42 \pm 6.34 \mathrm{vs}$ $33.38 \pm 5.96 \mathrm{ml} / 100 \mathrm{~g} / \mathrm{min} ; \mathrm{F}(1,27)=15.68, p<0.001)$. However, there were no main effects of PATCH or SMOKE, or any interactions, on gCBF among smokers. After controlling for sex, there was a trend towards less gCBF among smokers compared with nonsmokers (37.74 \pm 7.59 vs $43.14 \pm$ $10.80 \mathrm{ml} / 100 \mathrm{~g} / \mathrm{min} ; \mathrm{F}(1,57)=3.6, p=0.062)$, see Figure 2 .

\section{rCBF}

There was relatively little effect of the PATCH and SMOKE conditions on $\mathrm{rCBF}$. The main effect of PATCH revealed a single cluster of 176 voxels in the left temporal lobe ( $F$-value of peak voxel $=16.03$, MNI coordinates $-26,10,-35)$, related to greater $\mathrm{rCBF}$ in the PLA condition than in the NIC condition. No main effects of SMOKE or SMOKE $\times$ PATCH interactions were observed.

\section{Correlations between Withdrawal Symptoms and CBF}

Only the SJWQ subscales significantly affected by the SMOKE and PATCH conditions (craving, negative affect, and habit withdrawal) were included in the correlation analyses with global and regional CBF.

For the SMOKE condition (ABST-DENIC), change in craving was the only subscale that significantly correlated with change in $\operatorname{gCBF}(\mathrm{r}=0.40, p=0.035)$. None of the withdrawal subscales significantly predicted $\mathrm{gCBF}$ for the PATCH condition (PLA-NIC).

The changes in withdrawal scores and in $\mathrm{rCBF}$ for the SMOKE condition (ABST-DENIC) were entered into a multiple regression analysis. There were positive correlations between changes in craving scores and changes in rCBF throughout the frontal, parietal, and temporal cortices. These correlations also extended into the occipital cortex and cerebellum, see Figure 3 . In other words, the magnitude of change in craving symptoms from the denicotinized cigarette smoking condition to the abstinent condition corresponded to the magnitude of change in $\mathrm{rCBF}$ between conditions throughout several brain regions. Positive correlations were also found between reports of
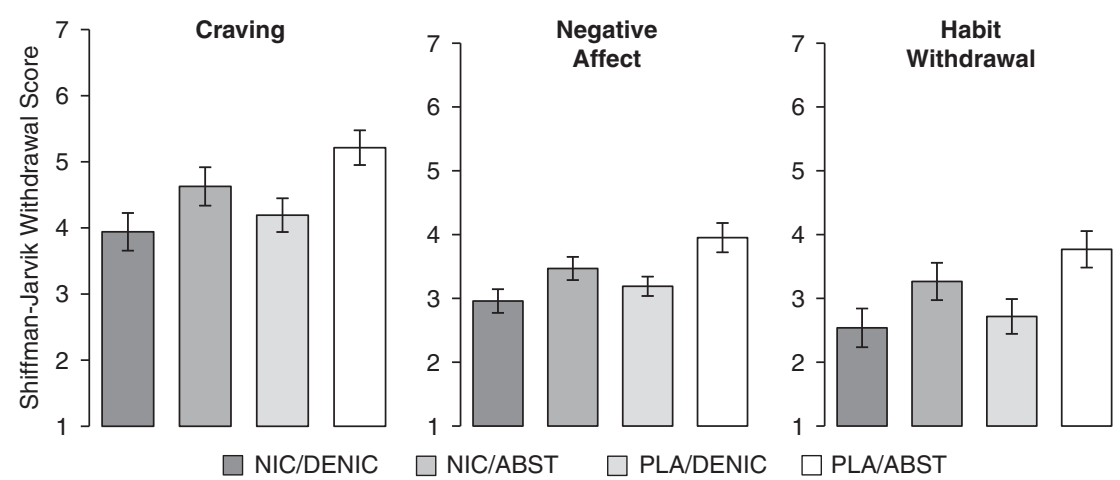

Figure I Self-reported symptoms from the Shiffman-Jarvik withdrawal questionnaire from each of the four experimental conditions. Scores were obtained after $24 \mathrm{~h}$ of exposure to each condition and immediately prior to the scanning session. Analyses of the subscales craving, negative affect, and habit withdrawal revealed main effects of PATCH (PLA vs NIC; $p<0.05$ ) and SMOKE conditions (ABST vs DENIC; $p<0.05$ ). Error bars are SEM. 
negative affect and $\mathrm{rCBF}$ in the right temporal lobe and in the left cerebellum. Habit withdrawal negatively correlated with $\mathrm{rCBF}$ in the cerebellum/brain stem, see Table 1.

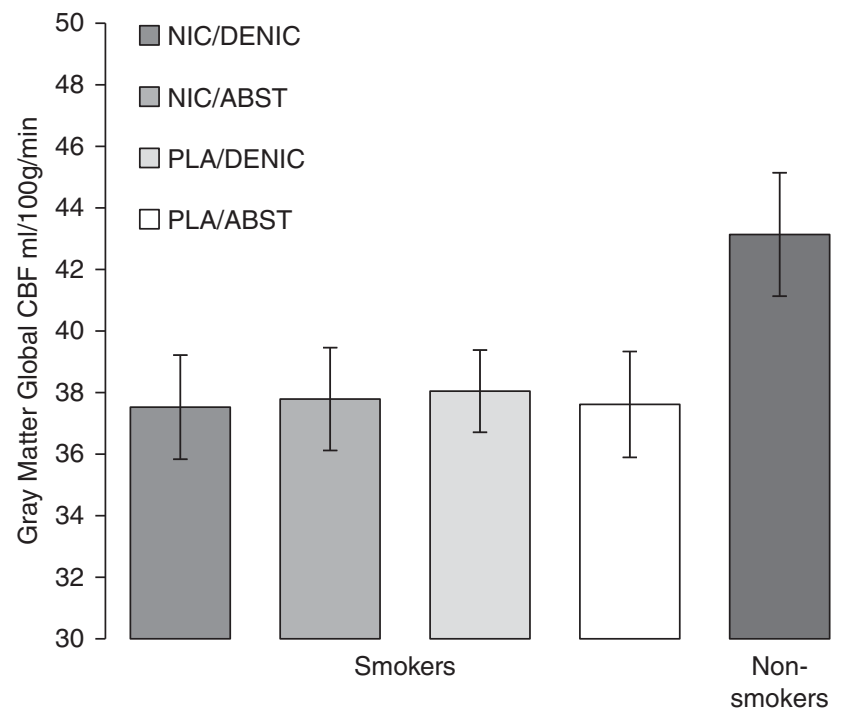

Figure 2 Whole-brain gray-matter global CBF. Shown are smokers' global CBF across the four conditions and nonsmokers' CBF from a single scan. There was no effect of PATCH or SMOKE conditions on smokers' CBF. On average, smokers had a trend towards less CBF than nonsmokers, controlling for sex $(p<0.07)$. Error bars are SEM.
Likewise, the changes in withdrawal scores and in $\mathrm{rCBF}$ between the PATCH conditions (PLA-NIC) were investigated. There was a positive correlation between craving scores and $\mathrm{rCBF}$ in the right occipital pole, and there were negative correlations between habit withdrawal scores and $\mathrm{rCBF}$ in the bilateral occipital cortex and the right cerebellum; see Table 2.

\section{DISCUSSION}

\section{Summary}

The current study evaluated the relationship between nicotine, cigarette smoking, and resting-state rCBF. Using a factorial design, we manipulated both nicotine and smoking conditions to isolate their main effects and/or interactions on $\mathrm{CBF}$. Consistent with prior reports, the effects of nicotine and non-nicotine smoking factors on global and regional CBF were minimal. However, changes in rCBF from the smoking to the abstinent conditions correlated with changes in self-reported craving and other withdrawal symptoms in parts of the frontal, parietal, occipital, temporal, and cerebellar cortices. Similar results have been reported previously; however, earlier studies were unable to separate nicotine from non-nicotine contributions to this relationship. Changes in withdrawal symptoms also correlated with changes in $\mathrm{rCBF}$ from the placebo patch to the nicotine patch conditions, albeit to a much lesser extent. Overall, the results of this study show that, after controlling

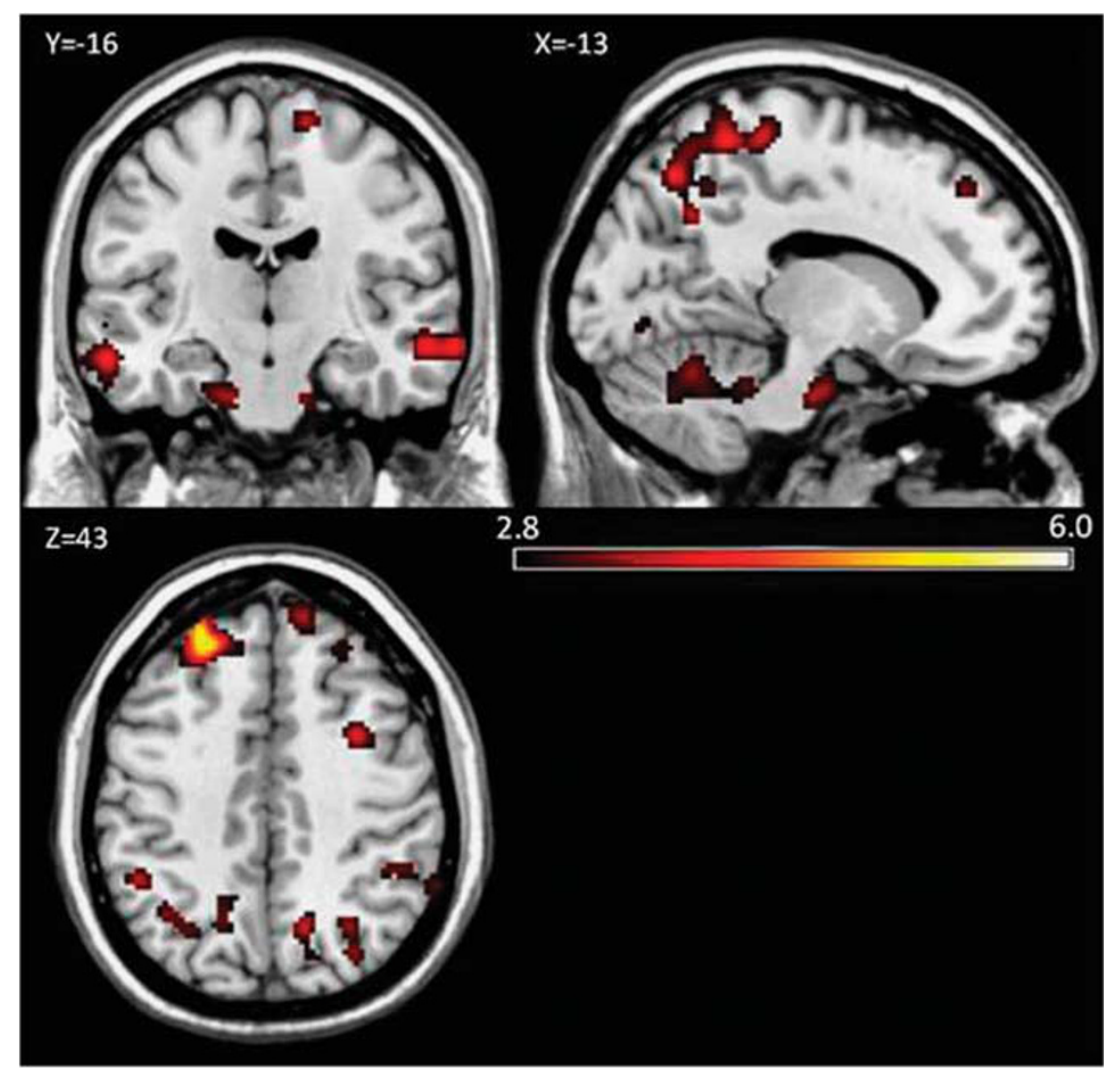

Figure 3 Statistical parametric maps of the correlation between changes in self-reported craving and changes in rCBF for the main effects of the SMOKE condition (ABST-DENIC). Positive correlations were found in frontal, parietal, occipital, temporal, and cerebellar cortices. Cluster corrected threshold $p<0.05$ ( $p<0.005$ uncorrected, $\geqslant 157$ contiguous voxels). Color bar indicates $t$-score values. 
Table I Regional Correlations Between Self-Reported Withdrawal Symptoms and CBF for the Main Effect of Smoke (ABST-DENIC)

\begin{tabular}{|c|c|c|c|c|}
\hline Brain region & Peak $x, y, z$ & Brodmann & T-max & $\mathbf{K}_{\mathbf{E}}$ \\
\hline \multicolumn{5}{|l|}{ Craving (positive) } \\
\hline L superior frontal gyrus/frontal pole & $-24,44,42$ & 9,46 & 5.95 & 588 \\
\hline $\mathrm{R}$ frontal pole/middle frontal gyrus & $36,40,24$ & $9,44,46$ & 4.05 & 314 \\
\hline $\mathrm{R}$ middle frontal gyrus & $32,8,41$ & $6,8,9,44$ & 4.03 & 202 \\
\hline R frontal pole & $10,50,40$ & 9 & 3.80 & 170 \\
\hline R postcentral \& precentral gyrus, precuneus cortex & $12,-44,63$ & $2,3,4,5,6,7$ & 4.89 & 3322 \\
\hline R lateral occipital cortex & $32,-72,39$ & 7,19 & 3.65 & 178 \\
\hline R temporal fusiform cortex/parahippocampal gyrus & $32,-6,-49$ & 20,36 & 5.65 & 756 \\
\hline $\mathrm{R}$ middle temporal gyrus & $66,-20,-13$ & $20,21,22$ & 4.55 & 374 \\
\hline L middle temporal gyrus & $-58,-14,-17$ & $20,21,22$ & 4.02 & 160 \\
\hline \multicolumn{5}{|l|}{ Negative affect (positive) } \\
\hline $\mathrm{R}$ middle temporal gyrus & $68,-16,-11$ & $20,21,22$ & 4.18 & 175 \\
\hline R parahippocampal gyrus & $30,-2,-33$ & 20,36 & 4.11 & 485 \\
\hline L cerebellum & $-10,-40,-29$ & & 3.74 & 648 \\
\hline \multicolumn{5}{|l|}{ Habit withdrawal (negative) } \\
\hline L brainstem/cerebellum & $-8,-28,-27$ & & 4.63 & 494 \\
\hline
\end{tabular}

Abbreviations: L, left, R, right.

Directions of the relationships are indicated as positive or negative. Cluster corrected threshold $p<0.05(p<0.005$ uncorrected, $\geqslant 157$-voxel cluster of contiguous significant voxels).

Table 2 Regional Correlations Between Self-Reported Withdrawal Symptoms and CBF for the Main Effect of Patch (PLA-NIC)

\begin{tabular}{lcccc}
\hline Brain region & Peak $\mathbf{x , y , z}$ & Brodmann & T-max & $\mathbf{K}_{\mathbf{E}}$ \\
\hline $\begin{array}{l}\text { Craving(positive) } \\
\text { R occipital pole }\end{array}$ & $18,-102,-1$ & 17 & 4.09 & 162 \\
& & & & \\
Habit withdrawal (negative) & & & & \\
R occipital pole & $20,-92,21$ & 18 & 4.44 & 233 \\
L occipital pole & $-26,-104,-7$ & 18 & 4.24 & 232 \\
R occipital fusiform gyrus & $26,-66,-13$ & 18,19 & 3.53 & 172 \\
R cerebellum & $24,-90,-31$ & & 4.13 & 167 \\
\hline
\end{tabular}

Abbreviations: L, left, R, right.

Directions of the relationships are indicated as positive or negative. Cluster corrected threshold $p<0.05$ ( $p<0.005$ uncorrected, $\geqslant 157$-voxel cluster of contiguous significant voxels).

for nicotine, the sensorimotor and non-nicotine psychoactive components of cigarette smoke significantly impact craving and associated brain regions. These results suggest there is increased activity in a fronto-temporo-cerebellar circuit that underlies the sensation of craving, and activity in this circuit is reduced by the act of smoking denicotinized cigarettes - in other words, sensations of craving and regional brain activity are suppressed by the non-nicotine psychoactive and sensorimotor components of tobacco smoke rather than by nicotine alone.

\section{Craving and Resting-State $\mathrm{CBF}$}

A previous study by Wang et al (2007), which used similar imaging methods, assessed CBF in smokers during smoking satiety and after $\geqslant 12 \mathrm{~h}$ of smoking abstinence. Although there was a limited effect of abstinence on $\mathrm{rCBF}$ and no effect on gCBF, the authors reported that abstinenceinduced craving correlated with abstinence-induced changes in $\mathrm{rCBF}$ in a number of cortical and subcortical areas; in particular, the thalamus, nucleus accumbens, temporal cortex, and prefrontal cortex. Other withdrawal symptoms, when combined into a single score, also correlated with $\mathrm{rCBF}$, although in fewer brain regions. They concluded that the correlations were primarily found in regions rich in $\alpha 4 \beta 2 \mathrm{nAChRs}$ linked with learning and memory and/or attention and behavioral control, and that these regions are specifically related to craving (rather than global withdrawal symptoms). Our study expanded on this research by separating the effects of nicotine from the act of smoking. Although we found few brain areas of overlap with Wang 
et al, the relationship we found between craving and $\mathrm{rCBF}$ from the ABST to the DENIC condition is unlikely to be caused by nicotine acting on its receptors (although denicotinized cigarettes contain trace amounts of nicotine, see limitations below).

Rather than being specific to actions at nicotine receptors, the measure of resting-state $\mathrm{rCBF}$ may indirectly reflect neural activity related to tobacco addiction and craving. In support of this, the change in craving scores from smoking denicotinized cigarettes to smoking usual brand cigarettes was found to negatively correlate with regional cerebral metabolic rate for glucose (rCMRglc) in the ventral striatum, orbitofrontal cortex, and pons (Rose et al, 2007). Another study showed a negative correlation between change in mood and ${ }^{11} \mathrm{C}$-raclopride binding potential before and after smoking a regular or denicotinized cigarette, indicating that greater dopamine release predicts more mood improvement (Brody et al, 2009b).

Among smokers, our results agree with previous studies showing correlations between subjective reports of withdrawal symptoms (including craving) and changes in $\mathrm{rCBF}$ (Franklin et al, 2007; Rose et al, 2003; Tanabe et al, 2008; Wang et al, 2007). Our results are also consistent with imaging studies using individuals addicted to drugs other than nicotine: Studies in which drug abusers are shown drug cues during a brain scan have reported increased activity in the dorsolateral prefrontal cortex, medial temporal lobe, and cerebellum among cocaine abusers (Grant et al, 1996), and increased activity in the inferior frontal cortex, fusiform gyrus, and cerebellum among heroin abusers (Xiao et al, 2006). The role that these regions have in working, episodic, and emotional memory suggests the fronto-temporo-cerebellar regions form a cognitive and emotional memory circuit that may underlie drug craving (Grant et al, 1996). The role of the cerebellum in addiction is poorly understood, but evidence suggests that the cerebellum contributes to executive function and inhibitory control (Bellebaum and Daum, 2007; Hester and Garavan, 2004; Rapoport et al, 2000). Our results indicate that increased brain activity in these regions is associated with strong drug cravings, which may reflect an enhanced cognitive-emotional memory circuit that promotes the anticipation of cigarette smoking.

\section{Nicotine Vs Non-Nicotine Components of Tobacco Withdrawal}

Increasing data support a critical role for non-nicotine psychoactive and sensorimotor effects in tobacco reinforcement and dependence. For instance, there is evidence that other components of tobacco smoke, such as the condensation products of acetaldehyde, have reinforcing properties and probably contribute to tobacco's addictive potential (Talhout et al, 2007). However, the contributions of nicotine and non-nicotine components to withdrawal symptoms are not well understood. Smoking denicotinized cigarettes can reduce cravings as much as nicotinized cigarettes (Domino et al, 2013), and a nicotine patch in the absence of smoking has been shown to have a limited, but significant, effect on reducing cravings and other withdrawal symptoms (Levin et al, 1994; Rose et al, 2001; Shiffman et al, 2006). This begs the question: are there identifiable differences between cravings for nicotine and cravings for the non-nicotine components of cigarettes? If so, are there withdrawal symptoms specific to the non-nicotine components of cigarettes that can be addressed to assist cessation?

In the current study, only three subscales of the SJWS were affected by the Patch and Smoke conditions, but all three of these subscales increased from the DENIC to the ABST conditions and from the NIC to the PLA conditions (albeit less robustly). Thus, we cannot infer the differential effects of nicotine or the non-nicotine components of smoking on these withdrawal subscales. A closer examination of the SJWQ subscales supports this inference. The questions regarding craving are broad (eg, Do you have an urge to smoke a cigarette? Are you thinking of cigarettes), and responses could be related to either the nicotine or non-nicotine components of cigarette smoking. Likewise, responses to the negative affect subscale (eg, Do you feel tense? Do you feel irritable?) could be due to both the nicotine and non-nicotine components of smoking as well, as both components are known to have psychoactive properties that affect mood. On the other hand, questions in the habit withdrawal subscale (eg, Do you miss having something to do with your hands? Do you miss inhaling cigarette smoke?) are specific to the sensorimotor aspects of smoking, yet subjects reported a reduction in habit withdrawal while wearing the nicotine patch during abstinence. More research is needed to understand the relationship between these three subscales.

Unlike the relationship between $\mathrm{rCBF}$ and craving, the correlations we found between $\mathrm{rCBF}$, negative affect, and habit withdrawal had a very limited extent and should be interpreted with caution. It is possible that craving, negative affect, and habit withdrawal are all integrally related, and the different relationships strengths between $\mathrm{rCBF}$ and the three SJWQ subscales could be accounted for by differences between the subscales themselves. For instance, the negative affect and habit withdrawal subscales consist of fewer question items than craving, and negative affect and habit withdrawal had slightly lower scores (shown in Figure 1). Alternatively, if the absence of a relationship between $\mathrm{rCBF}$ and other subscales is valid, this suggests that 'craving' is a unique condition related to the act of smoking, but unrelated to other tobacco withdrawal symptoms.

A useful addition to this line of research would be a measure that could differentiate between nicotine and nonnicotine effects of tobacco abstinence on withdrawal symptoms and craving. Although there are several measures of nicotine and tobacco withdrawal symptoms currently available (West et al, 2006), the development of a self-report scale that can parse apart withdrawal symptoms related to nicotine $v s$ non-nicotine effects of tobacco is warranted.

\section{Effects of Smoking on CBF}

A number of previous studies have investigated the effects of tobacco smoking on CBF. Long-term cigarette smoking has been shown to reduce rCBF (Rogers et al, 1985; Yamashita et al, 1988), possibly by hypocapnia caused by small airway disturbances (Yamashita et al, 1988) or by impaired neurovascular coupling owing to structural changes in blood vessels (Boms et al, 2010). rCBF decreases with advancing age, and differences between smokers 
and nonsmokers become more apparent in later years (Yamashita et al, 1988). In agreement with this previous work, we found slightly lower gCBF levels among smokers compared with nonsmokers; this finding and a replication of previously observed sex differences (Gur and Gur, 1990) validate of our methods.

Acutely following cigarette smoking or nicotine administration, smokers have reduced global brain activity, reduced $\mathrm{CBF}$, and increased regional responses in the prefrontal cortex, thalamus, and visual cortex; however, decreased regional responses have also been reported (Domino et al, 2000, 2004; Mathew and Wilson, 1991; Rose et al, 2003, for review, see Storage and Brody, 2012). Some of these differences may depend on whether smokers were abstinent or satiated when scanned (Tanabe et al, 2008; Zubieta et al, 2005). For instance, nicotine administered following overnight abstinence in smokers reduced $\mathrm{rCBF}$ in the temporal cortex and amygdala and increased rCBF in the thalamus (Zubieta et al, 2001). However, compared with smoking a denicotinized cigarette, smoking a regular cigarette increased $\mathrm{rCBF}$ in the occipital cortex, cerebellum, and thalamus and decreased $\mathrm{rCBF}$ in the anterior cingulate, nucleus accumbens, amygdala, and hippocampus (Zubieta et al, 2005). These differences may be related to the various components of cigarette smoke, different densities of nicotinic receptors among brain regions, individual differences among subjects, or differences in study design and methodologies. In particular, the route of administration (eg, intravenous, intranasal, dermal absorption) affects nicotine pharmacokinetics and may not replicate the inhalation of nicotine from cigarette smoke.

\section{Limitations}

Our study is not without limitations. pASL is a popular CBF imaging modality because of its straightforward implementation and high tagging efficiency. However, pASL has a lower signal-to-noise ratio than other modalities such as pseudocontinuous arterial spin labeling (pCASL) (Wu et al, 2007). The use of pCASL could have improved our detection of regional CBF patterns, although pASL and pCASL have been shown to produce similar mean perfusion levels (Gevers et al, 2011). Also, we obtained 24 image slices, which are more slices than usually obtained in perfusion imaging. It is possible that the end slices did not receive adequate signal for perfusion estimation, and this could account for our low whole-brain gray matter estimates. Second, the Quest 3 brand of denicotinized cigarettes yields $0.05 \mathrm{mg}$ of nicotine, which can result in $26 \% \alpha 4 \beta 2 \mathrm{nAChR}$ occupancy (Brody et al, 2009a). This concentration of nicotine is significantly lower than regular cigarettes (which contain $1.2-1.4 \mathrm{mg}$ of nicotine and occupy about $88 \%$ of nACh receptors (Brody et al, 2006)); however, a $0.05 \mathrm{mg}$ concentration of nicotine may be enough to induce feelings of satiety and offset withdrawal symptoms. In the study by Brody and colleagues, no relationship was found between receptor occupancy and withdrawal symptoms, which suggests there may be non-nicotine sensorimotor factors that are partly responsible for the relief of withdrawal (2009). Third, although we confirmed smoking satiety and abstinence with $\mathrm{CO}$ levels, we do not have a measure of serum nicotine concentrations. The concentrations of nicotine within- and between-subjects could have provided additional information regarding the fluctuation of withdrawal symptoms. Fourth, we used a fixed dose of $21 \mathrm{mg} / \mathrm{d}$ for the nicotine patch. Although this is the recommended supplementary dose for regular smokers, it does not take into account between-subject variations in preferred dose. It may have been too strong of a dose for some subjects and too weak of a dose for others. Also, dermal nicotine absorption has different pharmacokinetics than smoke inhalation (Benowitz et al, 1997). Last, we do not have a 'smoking as usual' condition for comparison; we did not scan subjects after smoking their own brand of cigarettes. Subjects may have had different expectations for the denicotinized cigarettes than for their usual brand, which could have influenced their self-report withdrawal scores and brain responses. Future studies in which expectancies are also manipulated (as in Harrell and Juliano, 2012) are warranted.

\section{Implications for Treatment and Tobacco Regulation}

The findings from this study may help explain smoking relapse during nicotine replacement therapy, as nicotine replacement therapy does not substitute the sensorimotor or other non-nicotine psychoactive components of smoking. The development of electronic cigarettes as a form of nicotine replacement therapy should be addressed in future research because they partially replicate the sensorimotor aspects of smoking. Other novel approaches to help smokers quit include smoking denicotinized cigarettes prior to a cessation attempt. Our results are also consistent with the view that presenting denicotinized cigarettes concurrently with the nicotine patch decouples the usual reinforcing effects of rapid nicotine administration from the sensorimotor effects of smoking, thereby promoting extinction of the reinforcing value of these sensorimotor cues. Current research in our laboratory is investigating the effects of such an intervention on smoking behavior, brain function, and cessation success. However, denicotinized cigarettes deliver other psychoactive compounds that could reinforce smoking behavior and prolonging exposure to these chemicals could undermine a quit attempt. Furthermore, reducing nicotine in cigarettes to levels below an addiction threshold has been proposed as a component of future FDA tobacco regulation.

\section{FUNDING AND DISCLOSURE}

JER receives grant funding from Philip Morris USA, and is a consultant for Novartis, Targacept and Philip Morris International (PMI), and has a patent purchase agreement with PMI for nicotine inhalation technology. FJM is a Site PI on an investigator initiated grant (GRAND) from Pfizer The remaining authors declare no conflict of interest

\section{ACKNOWLEDGEMENTS}

We thank Dr Thomas Liu at UC San Diego for providing the MATLAB script for the quantification of CBF, and Dr Todd Harshbarger at the Duke/UNC Brain Imaging and Analysis Center for image processing support. This work was supported by NIH grant R01 DA023516-01 (PI: McClernon) and K01 DA033347 (PI: Addicott). 


\section{REFERENCES}

APA (2000). Diagnostic and statistical manual of mental disorders: DSM-IV-TR. American Psychiatric Association: Washington, DC.

Bellebaum C, Daum I (2007). Cerebellar involvement in executive control. Cerebellum 6: 184-192.

Benowitz NL, Zevin S, Jacob P (1997). Sources of variability in nicotine and cotinine levels with use of nicotine nasal spray, transdermal nicotine, and cigarette smoking. Brit $J$ Clin Pharmacol 43: 259-267.

Bevins RA, Palmatier MI (2004). Extending the role of associative learning processes in nicotine addiction. Behav Cogn Neurosci Rev 3: $143-158$.

Boms N, Yonai Y, Sandor M, Rosengarten B, Bornstein NM, Csiba L et al (2010). Effect of smoking cessation on visually evoked cerebral blood flow response in healthy volunteers. JVasc Res 47: 214-220.

Brody AL, Mandelkern MA, Costello MR, Abrams AL, Scheibal D, Farahi J et al (2009a). Brain nicotinic acetylcholine receptor occupancy: effect of smoking a denicotinized cigarette. Int $J$ Neuropsychopharmacol 12: 305-316.

Brody AL, Mandelkern MA, London ED, Olmstead RE, Farahi J, Scheibal D et al (2006). Cigarette smoking saturates brain alpha(4)beta(2) nicotinic acetylcholine receptors. Arch Gen Psychiatry 63: 907-915.

Brody AL, Mandelkern MA, Olmstead RE, Allen-Martinez Z, Scheibal D, Abrams AL et al (2009b). Ventral striatal dopamine release in response to smoking a regular $v s$ a denicotinized cigarette. Neuropsychopharmacology 34: 282-289.

Buchhalter AR, Acosta MC, Evans SE, Breland AB, Eissenberg T (2005). Tobacco abstinence symptom suppression: the role played by the smoking-related stimuli that are delivered by denicotinized cigarettes. Addiction 100: 550-559.

Domino EF, Minoshima S, Guthrie S, Ohl L, Ni LS, Koeppe RA et al (2000). Nicotine effects on regional cerebral blood flow in awake, resting tobacco smokers. Synapse 38: 313-321.

Domino EF, Ni LS, Domino JS, Yang W, Evans C, Guthrie S et al (2013). Denicotinized versus average nicotine tobacco cigarette smoking differentially releases striatal dopamine. Nicotine Tob Res 15: 11-21.

Domino EF, Ni LS, Xu YJ, Koeppe RA, Guthrie S, Zubieta JK (2004). Regional cerebral blood flow and plasma nicotine after smoking tobacco cigarettes. Prog Neuropsychopharmacol Biol Psychiatry 28: 319-327.

Donny EC, Houtsmuller E, Stitzer ML (2007). Smoking in the absence of nicotine: behavioral, subjective and physiological effects over 11 days. Addiction 102: 324-334.

Franklin TR, Wang Z, Wang J, Sciortino N, Harper D, Li Y et al (2007). Limbic activation to cigarette smoking cues independent of nicotine withdrawal: a perfusion fMRI study. Neuropsychopharmacology 32: 2301-2309.

Gevers S, van Osch MJ, Bokkers RP.H, Kies DA, Teeuwisse WM, Majoie CB et al (2011). Intra- and multicenter reproducibility of pulsed, continuous and pseudo-continuous arterial spin labeling methods for measuring cerebral perfusion. J Cerebr Blood Flow Metab 31: 1706-1715.

Gilbert DG, McClernon FJ, Rabinovich NE, Plath LC, Jensen RA, Meliska CJ (1998). Effects of smoking abstinence on mood and craving in men: influences of negative-affect-related personality traits, habitual nicotine intake and repeated measurements. Pers Individ Dif 25: 399-423.

Gilbert DG, McClernon FJ, Rabinovich NE, Plath LC, Masson CL, Anderson AE et al (2002). Mood disturbance fails to resolve across 31 days of cigarette abstinence in women. J Consult Clin Psychol 70: 142-152.

Grant S, London ED, Newlin DB, Villemagne VL, Liu X, Contoreggi C et al (1996). Activation of memory circuits during cue-elicited cocaine craving. Proc Natl Acad Sci USA 93: 12040-12045.
Gross J, Lee J, Stitzer ML (1997). Nicotine-containing versus denicotinized cigarettes: effects on craving and withdrawal. Pharmacol Biochem Behav 57: 159-165.

Gur RE, Gur RC (1990). Gender differences in regional cerebral blood-flow. Schizophr Bull 16: 247-254.

Harrell PT, Juliano LM (2012). A direct test of the influence of nicotine response expectancies on the subjective and cognitive effects of smoking. Exp Clin Psychopharmacol 20: 278-286.

Heatherton TF, Kozlowski LT, Frecker RC, Fagerstrom KO (1991). The fagerstrom test for nicotine dependence: a revision of the fagerstrom tolerance questionnaire. Br J Addict 86: 1119-1127.

Hendricks PS, Ditre JW, Drobes DJ, Brandon TH (2006). The early time course of smoking withdrawal effects. Psychopharmacology 187: 385-396.

Hester R, Garavan H (2004). Executive dysfunction in cocaine addiction: evidence for discordant frontal, cingulate, and cerebellar activity. J Neurosci 24: 11017-11022.

Hughes JR, Hatsukami D (1986). Signs and Symptoms of tobacco withdrawal. Arch Gen Psychiatry 43: 289-294.

Iida M, Iida H, Dohi S, Takenaka M, Fujiwara H (1998). Mechanisms underlying cerebrovascular effects of cigarette smoking in rats in vivo. Stroke 29: 1656-1665.

Killen JD, Fortmann SP (1997). Craving is associated with smoking relapse: findings from three prospective studies. Exp Clin Psychopharmacol 5: 137-142.

Kim SG (1995). Quantification of relative cerebral blood-flow change by flow-sensitive alternating inversion-recovery (Fair) technique: application to functional mapping. Magn Reson Med 34: 293-301.

Levin ED, Westman EC, Stein RM, Carnahan E, Sanchez M, Herman S et al (1994). Nicotine skin patch treatment increases abstinence, decreases withdrawal symptoms, and attenuates rewarding effects of smoking. J Clin Psychopharmacol 14: 41-49.

Mathew RJ, Wilson WH (1991). Substance abuse and cerebral blood flow. Am J Psychiatry 148: 292-305.

McClernon FJ, Westman EC, Rose JE, Lutz AM (2007). The effects of foods, beverages, and other factors on cigarette palatability. Nicotine Tob Res 9: 505-510.

Perkins KA, Gerlach D, Vender J, Grobe J, Meeker J, Hutchison S (2001). Sex differences in the subjective and reinforcing effects of visual and olfactory cigarette smoke stimuli. Nicotine Tob Res 3: 141-150.

Pickworth WB, Fant RV, Nelson RA, Rohrer MS, Henningfield JE (1999). Pharmacodynamic effects of new de-nicotinized cigarettes. Nicotine Tob Res 1: 357-364.

Piper ME, Bolt DM, Kim SY, Japuntich SJ, Smith SS, Niederdeppe J et al (2008). Refining the tobacco dependence phenotype using the Wisconsin Inventory of Smoking Dependence Motives. J Abnorm Psychol 117: 747-761.

Rapoport M, van Reekum R, Mayberg H (2000). The role of the cerebellum in cognition and behavior: a selective review. J Neuropsychiatry Clin Neurosci 12: 193-198.

Rodgman A, Perfetti TA (2013). The chemical components of tobacco and tobacco smoke. 2nd edn, CRC press. Taylor and Francis Group: Boca Raton, FL.

Rogers RL, Meyer JS, Judd BW, Mortel KF (1985). Abstention from cigarette-smoking improves cerebral perfusion among elderly chronic smokers. JAMA 253: 2970-2974.

Rose JE (2006). Nicotine and nonnicotine factors in cigarette addiction. Psychopharmacology 184: 274-285.

Rose JE, Behm FM (2004). Extinguishing the rewarding value of smoke cues: pharmacological and behavioral treatments. Nicotine Tob Res 6: 523-532.

Rose JE, Behm FM, Salley AN, Bates JE, Coleman RE, Hawk TC et al (2007). Regional brain activity correlates of nicotine dependence. Neuropsychopharmacology 32: 2441-2452.

Rose JE, Behm FM, Westman EC (2001). Acute effects of nicotine and mecamylamine on tobacco withdrawal symptoms, cigarette 
reward and ad lib smoking. Pharmacol Biochem Behav 68: 187-197.

Rose JE, Behm FM, Westman EC, Johnson M (2000). Dissociating nicotine and nonnicotine components of cigarette smoking. Pharmacol Biochem Behav 67: 71-81.

Rose JE, Behm FM, Westman EC, Mathew RJ, London ED, Hawk TC et al (2003). PET studies of the influences of nicotine on neural systems in cigarette smokers. Am J Psychiatry 160: 323-333.

Rose JE, Levin ED (1991). Inter-relationships between conditioned and primary reinforcement in the maintenance of cigarette smoking. Br J Addict 86: 605-609.

Rose JE, Levin ED, Behm FM, Adivi C, Schur C (1990). Transdermal nicotine facilitates smoking cessation. Clin Pharmacol Ther 47: 323-330.

Shiffman S, Ferguson SG, Gwaltney CJ, Balabanis MH, Shadel WG (2006). Reduction of abstinence-induced withdrawal and craving using high-dose nicotine replacement therapy. Psychopharmacology 184: 637-644.

Shiffman SM, Jarvik ME (1976). Smoking withdrawal symptoms in two weeks of abstinence. Psychopharmacology 50: 35-39.

Shinohara T, Nagata K, Yokoyama E, Sato M, Matsuoka S, Kanno I et al (2006). Acute effects of cigarette smoking on global cerebral blood flow in overnight abstinent tobacco smokers. Nicotine Tob Res 8: 113-121.

Sinha AK, Klein J, Schultze P, Weiss J, Weiss HR (1991). Cerebral regional capillary perfusion and blood-flow after carbonmonoxide exposure. J Appl Physiol 71: 1196-1200.

Storage SS, Brody AL (2012). Functional brain imaging of tobacco exposure in humans. J Addict Res Ther S2: 003 doi:10.4172/21556105.S2-003.

Talhout R, Opperhuizen A, van Amsterdam JG.C (2007). Role of acetaldehyde in tobacco smoke addiction. Eur Neuropsychopharmacol 17: 627-636.

Tanabe J, Crowley T, Hutchison K, Miller D, Johnson G, Du YP et al (2008). Ventral striatal blood flow is altered by acute nicotine but not withdrawal from nicotine. Neuropsychopharmacology 33: 627-633.

Wang Z, Faith M, Patterson F, Tang K, Kerrin K, Wileyto EP et al (2007). Neural substrates of abstinence-induced cigarette cravings in chronic smokers. J Neurosci 27: 14035-14040.

Ward B (2000). Simultaneous inference for fMRI data. Biophysics Research Institute, Medical College of Wisconsin: Milwaukee.

Watkins SS, Koob GF, Markou A (2000). Neural mechanisms underlying nicotine addiction: acute positive reinforcement and withdrawal. Nicotine Tob Res 2: 19-37.

West R, Ussher M, Evans M, Rashid M (2006). Assessing DSM-IV nicotine withdrawal symptoms: a comparison and evaluation of five different scales. Psychopharmacology 184: 619-627.

Wong EC, Buxton RB, Frank LR (1998). Quantitative imaging of perfusion using a single subtraction (QUIPSS and QUIPSS II). Magn Reson Med 39: 702-708.

Wu WC, Fernandez-Seara M, Detre JA, Wehrli FW, Wang J (2007). A theoretical and experimental investigation of the tagging efficiency of pseudocontinuous arterial spin labeling. Magn Reson Med 58: 1020-1027.

Xiao ZW, Lee T, Zhang JX, Wu QL, Wu RH, Weng XC et al (2006). Thirsty heroin addicts show different fMRI activations when exposed to water-related and drug-related cues. Drug Alcohol Depend 83: 157-162.

Yamashita K, Kobayashi S, Yamaguchi S, Kitani M, Tsunematsu T (1988). Effect of smoking on regional cerebral blood-flow in the normal aged volunteers. Gerontology 34: 199-204.

Zubieta JK, Heitzeg MM, Xu YJ, Koeppe RA, Ni LS, Guthrie S et al (2005). Regional cerebral blood flow responses to smoking in tobacco smokers after overnight abstinence. Am J Psychiatry 162: $567-577$.

Zubieta JK, Lombardi U, Minoshima S, Guthrie S, Ni LS, Ohl LE et al (2001). Regional cerebral blood flow effects of nicotine in overnight abstinent smokers. Biol Psychiatry 49: 906-913. 